\title{
Hypoxia Induces Mesenchymal Gene Expression in Renal Tubular Epithelial Cells: An in vitro Model of Kidney Transplant Fibrosis
}

\author{
Stephanie Zella, b Roland Schmitt ${ }^{c}$ Sandra Witting ${ }^{a, b}$ Hans H. Kreipe ${ }^{a}$ \\ Kais Hussein ${ }^{a}$ Jan U. Becker ${ }^{a, b}$ \\ ${ }^{a}$ Institute of Pathology, ${ }^{b}$ Integrated Research and Treatment Center Transplantation (IFB-Tx), \\ and ${ }^{\mathrm{C}}$ Department of Nephrology and Hypertension, Hannover Medical School, Hannover, \\ Germany
}

Key Words

Hypoxia · Fibrosis · Matrix metalloproteinase- $2 \cdot \operatorname{miR}-124$

\begin{abstract}
Background: The development of interstitial fibrosis and tubular atrophy is a common complication after kidney transplantation and is associated with reduced long-term outcome. The hallmark of tubulointerstitial fibrosis is an increase in extracellular matrix resulting from exaggerated activation of fibroblasts/myofibroblasts, and tubular atrophy is characterized by a decrease in tubular diameter and loss of function. Atrophic epithelial cells may undergo epithelial-to-mesenchymal transition (EMT) with potential differentiation into interstitial fibroblasts. One potential driver of EMT in developing interstitial fibrosis and tubular atrophy is chronic hypoxia. Methods: The expression of 46 EMT-related genes was analyzed in an in vitro hypoxia model in renal proximal tubular epithelial cells (RPTEC). Furthermore, the expression of 342 microRNAs (miR) was evaluated in hypoxic culture conditions. Results: Hypoxic RPTEC expressed markers of a more mesenchymal phenotype and showed an increased expression of matrix metalloproteinase-2 (MMP2). MMP2 expression in RPTEC correlated inversely with a decreased expression of miR-124, which was found to have a putative binding site for the MMP2 transcript. Overexpression of miR-124 inhibited MMP2 protein translation. Hypoxia was associated with increased migration/proliferation of RPTEC which was reversed by miR-124. Conclusions: These results indicate that hypoxia promotes a mesenchymal and migratory phenotype in renal epithelial cells, which is associated with increased MMP2 expression. Hypoxia-dependent MMP2 expression is regulated via a reduced transcription of miR-124. Overexpression of miR-124 antagonizes hypoxia-induced cell migration. Further research is needed to elucidate the functional role of miR-124 and MMP2 in the development of fibrosis in renal transplant degeneration.




\section{Introduction}

The development of interstitial fibrosis and tubular atrophy is a common pathological process in kidney diseases and is also associated with chronic renal transplant degeneration. Interstitial fibrosis and tubular atrophy in renal transplants can be caused by ischemia/ hypoxia, reperfusion injury and immunological processes that lead to a progressive loss of functional transplant compartments. During the development of fibrosis, highly differentiated kidney parenchymal cells are replaced by scarring connective tissue and extracellular matrix components such as laminin, collagen and fibronectin [1]. An increase in the number and activity of extracellular matrix-producing myofibroblasts is a key element of this process. It has been suggested that approximately one third of myofibroblasts are not preexisting local fibroblasts but are a result of epithelial-to-mesenchymal transition (EMT). During the transformation process of EMT, epithelial cells activate genetic programs which result in the loss of epithelial markers (E-cadherin and cytokeratins) and upregulation of mesenchymal markers [vimentin, smooth muscle actin and S100 calcium binding protein A4 (S100A4)], loss of cell-cell basement membrane contact and phenotypic changes leading finally to spindleshaped myofibroblasts [2-4].

Several studies have implicated a role of microRNAs ( $\mathrm{miR}$ ) in the development of fibrosis and EMT. MicroRNAs are small ( 22 nucleotides) noncoding RNAs which regulate protein expression by posttranscriptional binding to the $3^{\prime}$-untranslated region ( $3^{\prime}$-UTR) of a corresponding mRNA. Base pairing of the $5^{\prime}$-microRNA nucleotides $2-8$, the so-called seed sequence, with target $3^{\prime}$-UTR is sufficient and essential for the microRNA function to induce translational repression or cleavage of target mRNA with subsequent degradation [5, 6]. Members of the miR-200 family (miR-200a, miR-200b, miR-200c, miR-141 and miR-429) and miR-205 are downregulated in TGF- $\beta$-induced EMT in canine kidney cells in vitro [7]. In a salt-induced hypertension and renal injury rat model, miR-29b has been shown to be upregulated in vivo and can regulate fibrosis-associated genes, particularly collagens and matrix metalloproteinase-2 (MMP2) in vitro [8]. These current findings, which are derived mainly from nonhuman cells, show that fibrotic processes in the kidney are regulated by microRNA.

Since hypoxia is thought to have a major impact on renal transplant fibrosis and can also induce EMT [9], it was the aim of this study to investigate the regulation of EMT-related genes and microRNAs in an in vitro hypoxia model in human renal proximal tubular cells and renal transplant biopsies.

\section{Subjects and Methods}

\section{Cell Culture and Hypoxia Assay}

Two types of cells were analyzed: primary nonneoplastic human renal proximal tubular epithelial cells (RPTEC; Lonza, Cologne, Germany) and human-derived 293 embryonic renal cells (DSMZ, Braunschweig, Germany). RPTEC were cultured in renal epithelial cell medium supplemented with REGM BulletKit (Lonza) according to the manufacturer's instructions. All cells were cultured at $37^{\circ} \mathrm{C}$ in a humidified atmosphere of $5 \% \mathrm{CO}_{2}$. Cells were used between passages 3 and 6. 293 cells were cultured with standard RPMI supplemented with 1\% L-glutamine, $10 \%$ fetal calf serum and $1 \%$ antibiotics.

Hypoxic culture conditions were used for RPTEC and were achieved by using the Don Whitley H35 Hypoxystation (Don Whitley Scientific Ltd., Shipley, UK). RPTEC were seeded at $1 \times 10^{6}$ cell/T25 flask 1 day prior to the experiment to become adherent. Cells were maintained at $1 \% \mathrm{O}_{2}$ for $48 \mathrm{~h}$. 


\section{MicroRNA Transfection}

Cells were plated in a $6-\mathrm{cm}$ dish $6 \mathrm{~h}$ prior to transfection. For each experiment $100 \mathrm{~nm}$ of pre-miR-124 precursor molecules or scrambled microRNA control molecules were used. Two different transfection systems were used, one for each cell type as recommended by the manufacturers. RPTEC $\left(1 \times 10^{6}\right)$ were transfected using PrimeFect Reagent (Lonza) according to the manufacturer's protocol. The 293 cells $\left(1.2 \times 10^{6}\right)$ were transfected with Lipofectamine LTX and Plus Reagent according to the manufacturer's protocol (Invitrogen, Life Technologies, Carlsbad, Calif., USA). Total RNA and protein lysates were prepared 4 days after transfection.

\section{Scratch Assay}

A scratch assay was performed in $80 \%$ confluent monolayer culture in 6-well plates. $1 \times$ $10^{6}$ RPTEC and 293 cells were seeded $48 \mathrm{~h}$ prior to scratching. A mean 1,250- $\mathrm{mm}$ (range $1,180-1,300 \mu \mathrm{m}$ ) cell-free zone was scratched using a standard 2-ml pipette. Afterwards, cells were washed twice to remove nonadherent cells. Inverted microscopic images were taken at 0 and $16 \mathrm{~h}$ after scratching using a CKX41 microscope and SC30 camera (both Olympus).

\section{Quantitative PCR for MicroRNA Expression Analysis}

Total RNA was isolated using TRIzol reagent (Invitrogen, Life Technologies) according to the manufacturer's protocol. TaqMan MicroRNA Assays were used to detect mature microRNAs with microRNA-specific looped RT primers and TaqMan probes as recommended by the manufacturer (Applied Biosystems, Life Technologies).

High-throughput microRNA screening was performed using TaqMan MicroRNA Array Panel encompassing 342 mature human microRNAs and 6 reference genes: U6 (4×), RNU44 and RNU48 (Applied Biosystems) according to the manufacturer's protocol. cDNA was analyzed by TaqMan MicroRNA Array in duplicate using the 7900 HT Fast Real-Time PCR system.

The expression of single microRNAs was analyzed using miR-qRT-PCR. PCR was performed in duplicate using the TaqMan 7500 System. microRNA expression was normalized relative to U6 expression, which was nearly equal under all conditions (data not shown), and quantified relative to control-treated cells by the $\Delta \Delta \mathrm{Ct}$ method [10].

\section{Quantitative PCR for EMT-Associated mRNA Expression Analysis}

A custom TaqMan Low Density Array (Applied Biosystems) was spotted with 46 genes related to EMT and 2 reference genes [RNA polymerase 2 (POLR2A) and $\beta$-glucuronidase (GUSB)] on a 384-well microfluidic card $(8 \times 48)$. The list of analyzed protein-coding genes is provided in online supplementary table 1 (for all online suppl. material, see www. karger.com/doi/10.1159/000351046). One microgram of total RNA was reverse transcribed using the High-Capacity cDNA Reverse Transcription Kit and Random Primer (Applied Biosystems) according to the manufacturer's protocol. Twenty microliters of cDNA, $30 \mu \mathrm{H}_{2} \mathrm{O}$ and $50 \mu \mathrm{l}$ TaqMan Gene Expression Master Mix (Applied Biosystems) were mixed and loaded on the array. TaqMan Low Density Arrays were performed on the 7900 HT Fast Real-Time PCR system using SDS 2.3 software. Four independent experiments were performed.

For the reevaluation of single genes, real-time PCR was performed using TaqMan Gene Expression Assays (Hs00234422_m1 MMP2,Hs00172187_m1 POLR2A and Hs99999908_m1 GUSB) and TaqMan Gene Expression Master Mix (all Applied Biosystems) according to the manufacturer's protocol. Data were analyzed by the $\Delta \Delta \mathrm{Ct}$ method [10] and GraphPad Prism 5 . Relative expression levels of all genes were calculated as 2 -[(Ct reference gene) - (Ct target gene)] $\left(2^{-\Delta C t}\right)$. 
Fig. 1. Gene expression of MMP2 in hypoxic RPTEC compared to control cells. Four independent experiments were performed, and relative expression was calculated as $2^{-\Delta \mathrm{Ct}}$ to the POLR2A reference gene. MMP2 is upregulated 2-fold in hypoxic renal epithelial cells. $w \mathrm{t}=$ Wild-type RPTEC.

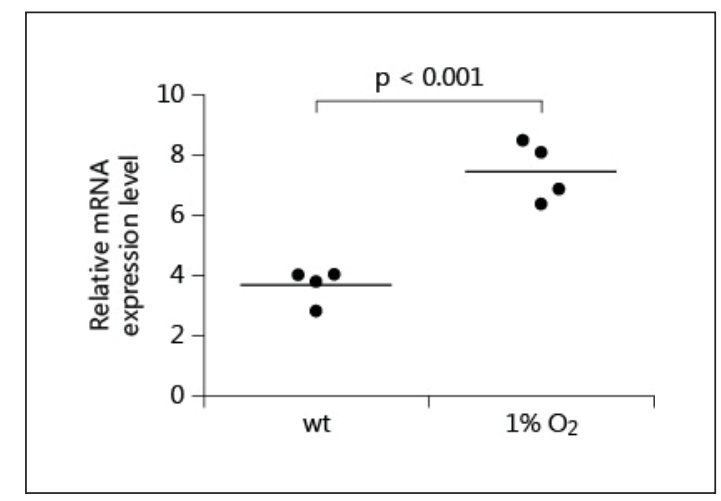

\section{Immunoblot for MMP2 Expression Analysis}

Cell lysates from miR-124-transfected 293 cells were prepared using lysis buffer (RIPA buffer: 50 mM Tris-HCl pH 7.5, 150 mM NaCl, 5 mm EDTA, 1\% Triton X-100, 0.5\% Na-deoxycholate, $0.1 \%$ sodium dodecyl sulfate and protease inhibitor cocktail Complete Mini; Roche, Basel, Switzerland). Proteins were separated by sodium dodecyl sulfate polyacrylamide gel electrophoresis and transferred to a Protran ${ }^{\circledR}$ nitrocellulose transfer membrane (Whatman, Maidstone, UK). After blocking with 5\% milk solution, the membrane was incubated with MMP2 (No. MA1-16641; Pierce, Thermo Scientific, Rockford, Ill., USA) and $\beta$-actin (No. ab6276; Abcam, Cambridge, UK) antibody according to the manufacturer's protocol.

ECL Western Blotting Substrate (Pierce) was used to detect horseradish peroxidase enzyme activity by chemiluminescence. Densitometry was performed using an LAS-1000 Imaging System (Fujifilm, Tokyo, Japan) and Image Reader LAS-1000 V1.11 software.

\section{Statistical Analysis}

All data are expressed as relative expression levels and calculated as $2^{-\Delta \mathrm{Ct}}$. The unpaired $\mathrm{t}$ test was employed using GraphPad Prism Software. Statistical significance was set at $\mathrm{p}<$ 0.05 .

\section{Results}

Hypoxia-Related mRNA and MicroRNA Expression in Human Renal Tubular Cells

Hypoxia-associated molecular changes were analyzed in RPTEC which had been incubated for $48 \mathrm{~h}$ at $1 \% \mathrm{O}_{2}$. Ten out of 46 protein-coding gene transcripts were significantly $(\mathrm{p}<$ 0.05) deregulated under hypoxic conditions. Upregulated genes included MMP2 (2-fold) (fig. 1a), serpine 1 (2.5-fold), integrin alpha 5 (ITGA5; 1.9-fold) and twist homolog 1 (Drosophila; TWIST; 4.2-fold) among others. Downregulated gene transcripts included signal transducing factor ras homolog gene family, member A (RHOA; 1.6-fold), connective tissue growth factor (CTGF; 2.4-fold) and mechanistic target of rapamycin (serine/threonine kinase; MTOR; 1.7-fold) (online suppl. table 1).

Despite failing to reach statistical significance, several typical mesenchymal genes showed increased transcript levels: vimentin (1.9-fold, $p=0.063)$, smooth muscle actin/ ACTA2 (1.4-fold, $p=0.094)$, S100A4 (1.5-fold, $p=0.51$ ) and collagen 1A2/COL1A2 (2.6-fold, $\mathrm{p}=0.099$ ). Epithelial genes E-cadherin/CDH1 and cytokeratin 19 were unchanged while cytokeratin 18 showed a trend for downregulation (online suppl. table 1). 
Fig. 2. Analysis of MMP2 for miR124 binding sites using RNA22. mRNA is shown in normal print, and miR-124 is shown in bold print. miR-124 targets MMP2 with complete seed match $\left(5^{\prime}\right.$ end) and favorable low energy.

Fig. 3. Representative Western blot of MMP2 in pre-miR-control (ctrl) and pre-miR-124-transfected 293 cells. The MMP2 protein level is reduced $\sim 30 \%$ in cells overexpressing miR-124 compared to control cells. Cells were analyzed 4 days after transfection and three independent experiments were performed.
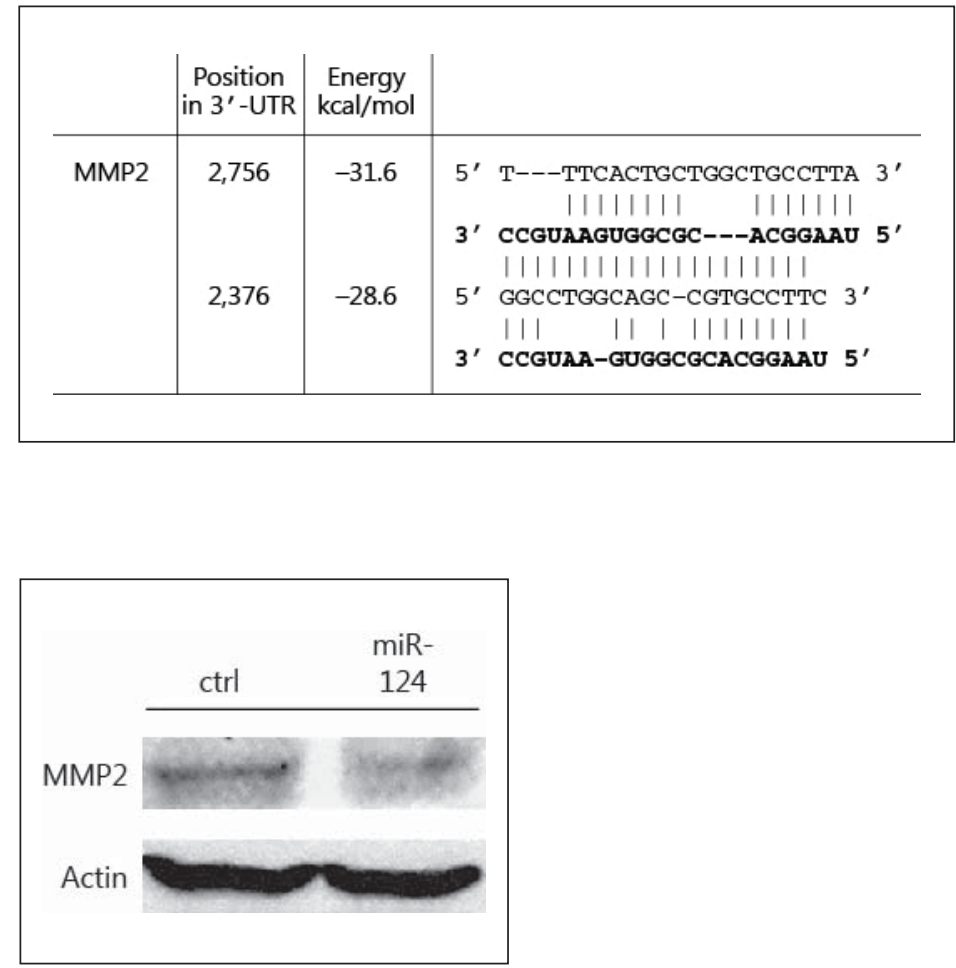

Analysis of the corresponding microRNA expression profile revealed expression of a total of 310/342 microRNAs and 43/310 of these microRNAs were expressed in a hypoxia-sensitive fashion (online suppl. table 2). Thirty-five microRNAs were upregulated (e.g. miR-200c and miR-21) and 8 were downregulated (e.g. miR-205 and miR-124).

Hypoxia-Induced miR-124 Expression Regulates MMP2 Expression in Renal Tubular Cells

We compared deregulated mRNA and microRNA and performed target prediction by using DIANA [11] and RNA22 [12] microRNA-target prediction tools. RNA22 revealed two potential miR-124 binding sites in MMP2 3'-UTR with favorable low energy of -28.6 and $-31.6 \mathrm{kcal} / \mathrm{mol}$ (fig. 2). In our experiments, hypoxia induced a 2-fold upregulation of MMP2 expression and 5-fold downregulation of miR-124 compared to normoxia in RPTEC.

For in vitro analysis of a potential regulation of MMP2 mRNA by miR-124, microRNA was transfected and MMP2 protein expression was evaluated. As MMP2 protein could not be sufficiently detected by Western blot in RPTEC, we alternatively used human-derived renal 293 cells for miR-124/MMP2 analysis. Four days after pre-miR-124 transfection, miR-124 was induced 400-fold in 293 cells compared to scrambled control cells. miR-124-overexpressing cells revealed a 25-35\% reduction of MMP2 protein expression, which indicates an miR124-dependent regulation of MMP2 (fig. 3).

\section{miR-124 Overexpression Restores Hypoxia-Induced Enhanced RPTEC Migration}

Because tubulointerstitial fibrosis is crucially based on migration of previously adherent cells, we performed scratch assays. Under normoxia, RPTEC have a low migration/proliferation capability. Sixteen hours after scratching, a 1,250 $\pm 70 \mu \mathrm{m}$ cell-free zone was reduced by $210 \pm 100 \mu \mathrm{m}(17 \pm 10 \%)$. Hypoxic culture conditions increased migration/proliferation of RPTEC to $\sim 2.5$-fold: cells migrated $530 \pm 100 \mu \mathrm{m}(42 \pm 10 \%)$ into the cell-free zone (fig. 4$)$. 


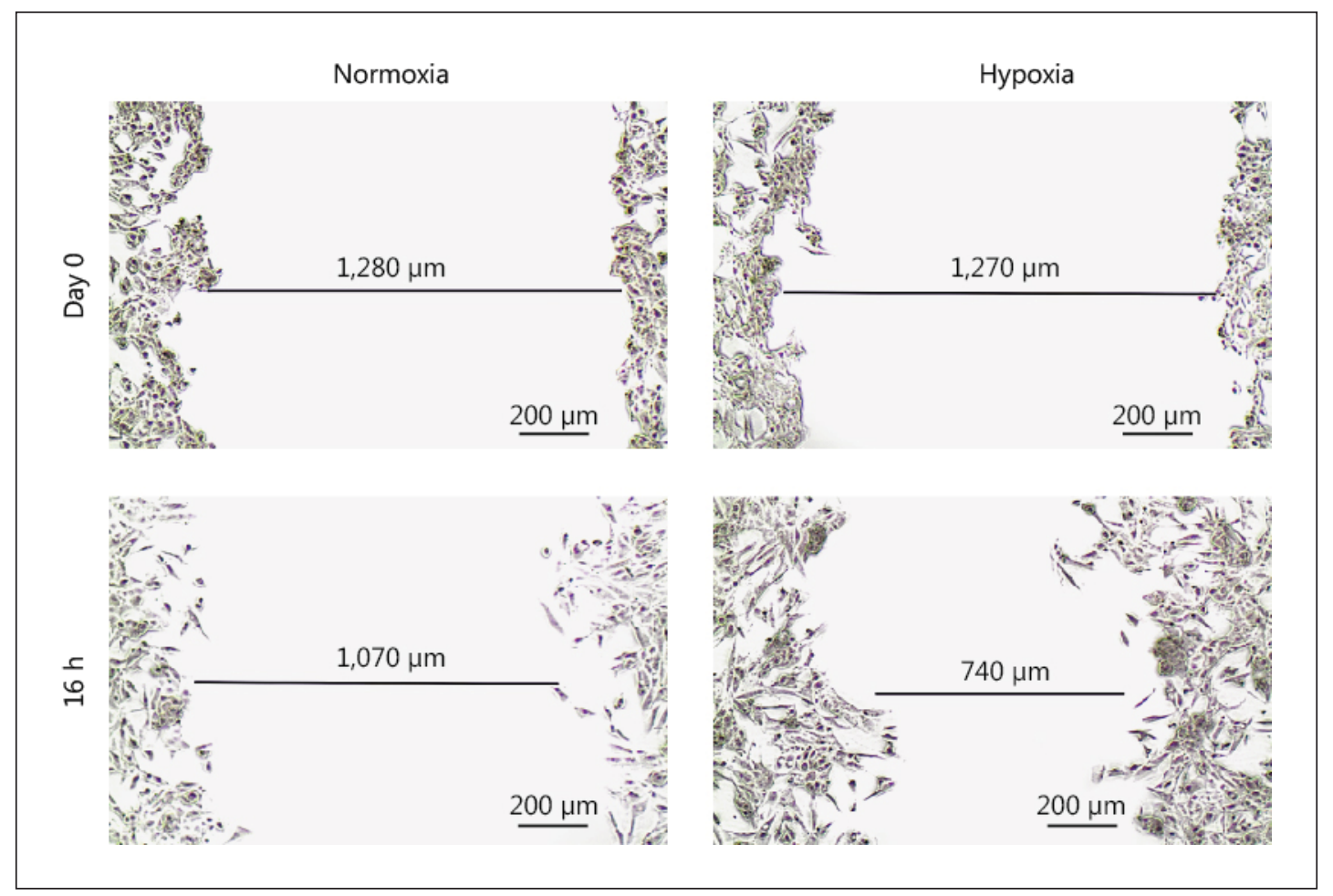

Fig. 4. Scratch assay. $80 \%$ confluent monolayer RPTEC were wounded with a pipette and incubated for $16 \mathrm{~h}$ with normal and low (1\%) $\mathrm{O}_{2}$ amounts. Hypoxia increased migration/proliferation capability of RPTEC 2.5fold.

Next, we wanted to evaluate a potential link between hypoxia-related downregulation of miR-124 and the increased migration/proliferation. A scratch assay was performed 3 days after transfection of RPTEC with scrambled control or with miR-124 precursor molecules, which resulted in an $\sim 10,000$-fold increased expression of mature miR-124 compared to controls. Reepithelization was monitored after $16 \mathrm{~h}$ under hypoxia and revealed that control cells (transfected with scrambled control microRNA) grew $490 \pm 90 \mu \mathrm{m}(46 \pm 6 \%)$ into the cell-free zone. In contrast, under hypoxia, miR-124-overexpressing cells migrated only $160 \pm$ $30 \mu \mathrm{m}(15 \pm 3 \%)$, showing that miR-124 antagonizes hypoxia-induced migration (fig. 5).

\section{Discussion}

A key initiating factor of chronic transplant fibrosis is hypoxia. The molecular pathway is directed through hypoxia-inducible factor 1 alpha (HIF-1 $\alpha$ ) signaling by induction of lysyl oxidase genes [9]. As a molecular indicator of hypoxia response, we found an upregulation of the oxygen-sensitive genes GAPDH, serpine 1 and TWIST1 in renal cells in vitro $[13,14]$. TWIST1 is induced via HIF-1 $\alpha$ and has previously been shown to be increased in hypoxic renal cells in vitro [15], and we also found increased levels of this hypoxia-associated factor in hypoxic renal epithelial cells. Similar to previous data, in our experiments HIF-1 $\alpha$ transcripts were not increased by low oxygen because HIF- $1 \alpha$ is regulated on the protein and not the transcript level $[16,17]$. 
Zell et al.: Hypoxia Induces Mesenchymal Gene Expression in Renal Tubular Epithelial Cells: An in vitro Model of Kidney Transplant Fibrosis

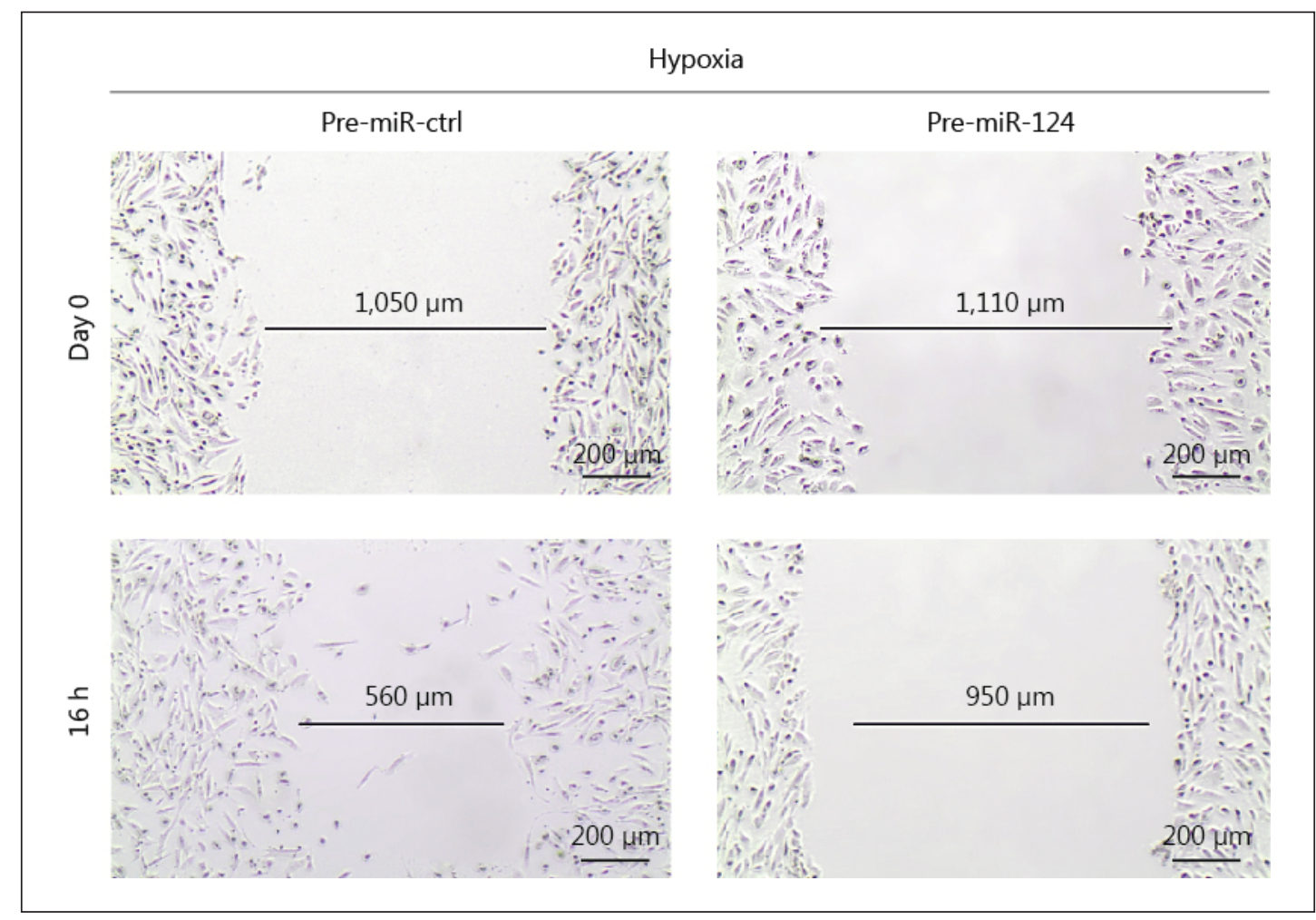

Fig. 5. Scratch assay in transfected RPTEC in hypoxic cell culture condition. Pre-miR-124 restores an increased hypoxic migration/proliferation of RPTEC to a normal level $16 \mathrm{~h}$ after scratching. $\mathrm{ctrl}=\mathrm{Control}$.

Renal epithelial cells are normally highly differentiated, which is crucial for adequate vectorial transport function. They have a low proliferative rate and are sessile with no need for migration. Following acute cell loss during kidney injury, surviving tubular epithelial cells undergo a transient process of dedifferentiation in which they express mesenchymal markers such as vimentin, ACTA2 and S100A4 [18]. This dedifferentiation enables the cells to proliferate and migrate, which is necessary for the replacement of lost cells [19]. Subsequently, the dedifferentiated cells must redifferentiate back to an epithelial phenotype. If this process is disrupted, the sustained dedifferentiation may promote tubular atrophy, fibrosis and scarring [18]. Alternatively, dedifferentiated cells might further degenerate into a full mesenchymal phenotype, including a transit from the tubular microenvironment into the interstitial space, i.e. EMT. In 2002, Rastaldi et al. [20] studied EMT in a set of 133 renal biopsies and identified the majority of tubular cells as being double-positive for mesenchymal markers and cytokeratins. However, experimental evidence of EMT in vivo remains controversial [21,22] and its demonstration is challenging. As indicated by our experiments, EMT, as defined by a loss of epithelial marker gene expression and an expression of mesenchymal genes [3], could not be completely induced in hypoxic renal epithelial cells in vitro. However, we found a marker expression profile of functional epithelial dedifferentiation including the decreased cytokeratin 18 and the increased mesenchymal genes vimentin, ACTA2, COL1A2 and S100A4.

A hypoxia-induced expression of MMP2 has previously also been shown in human endothelial cells [23]. MMP2 cleaves type IV collagen, the main component of basement membranes, and increased MMP2 expression is associated with moderate and severe tubulointerstitial damage [24]. Recent data underline the crucial role that MMP2 might play in renal fibrosis by 
inducing epithelial dedifferentiation, migration and EMT [25]. Indeed, we found that, in addition to increased MMP2 expression, hypoxic renal cells have a higher migration/proliferation capability. We found a correlation between upregulated MMP2 expression and decreased levels of miR-124 and identified MMP2 as a direct target of miR-124. Functionally, overexpression of miR-124 reduced hypoxia-induced migration/proliferation in renal epithelial cells. MicroRNAs can inhibit mRNA/protein translation by semicomplementary binding to the target mRNA and can therefore regulate more than one gene. Thus, although we could show that miR-124 decreases MMP2 protein levels, it cannot be excluded that the miR-124-associated effect on migration/proliferation is linked to additional gene products. Other potential miR-124 targets are, for example, transcription factor v-ets erythroblastosis virus E26 oncogene homolog 1 (avian; ETS1) as well as SMAD family member 5 (SMAD5) and bone morphogenetic protein 6 (BMP6). In contrast to MMP2, the expression of these factors was not induced by hypoxia (no major changes of ETS1, SMAD5 and BMP6 levels).

In summary, hypoxia cannot induce complete EMT with loss of epithelial markers in renal tubular epithelial cells in vitro, but cells show elevated levels of mesenchymal gene transcripts including vimentin, ACTA2, S100A4 and COL1A2. Hypoxic renal epithelial cells have a higher migration/proliferation capability and show an increased expression of MMP2, which we identify as a new target of miR-124. Overexpression of miR-124 antagonizes increased migration/proliferation in hypoxia, but this effect might include additional target transcripts independent of MMP2. Further research is needed to functionally elucidate whether hypoxiainduced suppression of miR-124 and upregulation of MMP2 might contribute to renal allograft fibrosis, which could have implications for future therapeutic developments.

\section{Acknowledgements}

This work was supported by a grant from the German Federal Ministry of Education and Research (reference No. 01E00802). The contents of this article are the sole responsibility of the authors.

\section{Disclosure Statement}

The authors have no conflicts of interest to declare.

\section{References}

$>1$ Wynn TA: Common and unique mechanisms regulate fibrosis in various fibroproliferative diseases. J Clin Invest 2007;117:524-529.

2 Kalluri R, Neilson EG: Epithelial-mesenchymal transition and its implications for fibrosis. J Clin Invest 2003; 112:1776-1784.

3 Carew RM, Wang B, Kantharidis P: The role of EMT in renal fibrosis. Cell Tissue Res 2012;347:103-116.

4 Vongwiwatana A, Tasanarong A, Rayner DC, Melk A, Halloran PF: Epithelial to mesenchymal transition during late deterioration of human kidney transplants: the role of tubular cells in fibrogenesis. Am J Transplant 2005; 5:1367-1374.

5 Bartel DP: MicroRNAs: genomics, biogenesis, mechanism, and function. Cell 2004;116:281-297.

6 Lewis PB, Burge CB, Bartel DP: Conserved seed pairing, often flanked by adenosines, indicates that thousands of human genes are microRNA targets. Cell 2005;120:15-20.

7 Gregory PA, Bracken CP, Bert AG, Goodall GJ: MicroRNAs as regulators of epithelial-mesenchymal transition. Cell Cycle 2008; 7:3112-3118.

8 Liu Y, Taylor NE, Lu L, et al: Renal medullary microRNAs in Dahl salt-sensitive rats: miR-29b regulates several collagens and related genes. Hypertension 2010;55:974-982. 
- Higgins DF, Kimura K, Bernhardt WM, et al: Hypoxia promotes fibrogenesis in vivo via HIF-1 stimulation of epithelial-to-mesenchymal transition. J Clin Invest 2007;117:3810-3820.

10 Livak KJ, Schmittgen TD: Analysis of relative gene expression data using real-time quantitative PCR and the 2(-Delta Delta C(T)) method. Methods 2001;25:402-408.

11 Vlachos IS, Kostoulas N, Vergoulis T, et al: DIANA miRPath v2.0: investigating the combinatorial effect of microRNAs in pathways. Nucleic Acid Res 2012;40:W498-W504.

12 Miranda KC, Huynh T, Tay Y, et al: A pattern-based method for the identification of MicroRNA binding sites and their corresponding heteroduplexes. Cell 2006;126:1203-1217.

13 Graven KK, McDonald RJ, Farber HW: Hypoxic regulation of endothelial glyceraldehyde-3-phosphate dehydrogenase. Am J Physiol 1998;274:C347-C355.

14 Uchiyama T, Kurabayashi M, Ohyama Y, et al: Hypoxia induces transcription of the plasminogen activator inhibitor-1 gene through genistein-sensitive tyrosine kinase pathways in vascular endothelial cells. Arterioscler Thromb Vasc Biol 2000;4:1155-1161.

$\checkmark 15$ Sun S, Ning X, Zhang Y, et al: Hypoxia-inducible factor-1 $\alpha$ induces twist expression in tubular epithelial cells subjected to hypoxia, leading to epithelial-to-mesenchymal transition. Kidney Int 2009;75:1278-1287.

16 Wenger RH, Kvietikova I, Rolfs A, Gassmann M, Marti HH: Hypoxia-inducible factor-1alpha is regulated at the post-mRNA level. Kidney Int 1997;51:560-563.

17 Uchida T, Rossignol F, Matthay MA, et al: Prolonged hypoxia differentially regulates hypoxia-inducible factor (HIF)-1alpha and HIF-2alpha expression in lung epithelial cells. J Biol Chem 2004;279:14871-14878.

18 Ishibe S, Cantley LG: Epithelial-mesenchymal-epithelial cycling in kidney repair. Curr Opin Nephrol Hypertens 2008;4:379-385.

19 Bonventre JV, Yang L: Cellular pathophysiology of ischemic acute kidney injury. J Clin Invest 2011;121:4210 4221.

20 Rastaldi MP, Ferrario F, Giardino, et al: Epithelial-mesenchymal transition of tubular epithelial cells in human renal biopsies. Kidney Int 2002;62:137-146.

-21 Fragiadaki M, Mason RM: Epithelial-mesenchymal transition in renal fibrosis - evidence for and against. Int J Exp Pathol 2011;92:143-150.

-22 Barnes JL, Glass II WF: Renal interstitial fibrosis: a critical evaluation of the origin of myofibroblasts. Contrib Nephrol 2011;169:73-93.

23 Ben-Yosef Y, Miller A, Shapiro S, Lahat N: Hypoxia of endothelial cells leads to MMP-2-dependent survival and death. Am J Physiol Cell Physiol 2005;289:C1321-C1331.

24 Aresu L, Benali S, Garbisa S, Gallo E, Castagnaro M: Matrix metalloproteinases and their role in the renal epithelial mesenchymal transition. Histol Histopathol 2011;26:307-313.

-25 Du X, Shimizu A, Masuda Y, et al: Involvement of matrix metalloproteinase-2 in the development of renal interstitial fibrosis in mouse obstructive nephropathy. Lab Invest 2012;92:1149-1160. 